\title{
Optothermal detection of subsurface graphical features in artworks
}

\author{
by S.Paoloni * , F.Mercuri, N.Orazi, C.Cicero, U.Zammit \\ * Dipartimento di Ingegneria Industriale, Università degli studi di Roma "Tor Vergata", Rome, Italy, \\ stefano.paoloni@uniroma2.it
}

\begin{abstract}
A method based on the use of active infrared thermography for the identification of hidden graphical features is here presented. A theoretical model of the thermographic signal is proposed where the main physical mechanisms allowing the identification of the buried features are described. Such a model is then adopted for the analysis of the results obtained on specifically designed test samples.
\end{abstract}

\section{Introduction}

Nondestructive inspection techniques are increasingly used in the field of cultural heritage since these allow to obtain valuable information about artworks, such as those concerning their preservation state or their manufacturing process. Among others, the techniques based on the use of infrared (IR) radiation are widely adopted in this field [1,2]. In particular, infrared reflectography (IRR) [3] is routinely employed for the inspection of paintings and, in particular, for the detection of drawings buried below the paint layer. Such an application makes use of the optical contrast offered by reflectograms obtained by recording the reflected component of the radiation illuminating the painting in the near infrared spectral range (NIR: 0.7-2.5 $\mu \mathrm{m}$ ). In fact, NIR radiation can penetrate through the paint layers more easily than the visible one (VIS), and, hence, it can reach the ground where it can be either reflected back or absorbed by IR absorbing materials such as graphite. Recently, a new reflectographic configuration [4] based on the detection of the IR radiation in the medium wavelength range (MWIR: 3-5 $\mu \mathrm{m}$ ) has also been proposed. Regardless of the adopted IR wavelength range, one of the main limiting factors of reflectography is that it does not provide any quantitative information about the depth of the detected features.

Active infrared thermography (IRT) is another well established tool for the nondestructive inspection of artworks [5-7]. Such a technique makes use of the contrast in the recorded thermograms originated by the presence of subsurface thermal inhomogeneities [8]. Recently, the authors have successfully adopted pulsed IRT in the MWIR range for detection of texts buried beneath the end papers of bookbindings of ancient volumes [9] and, in addition, of underdrawings below the pigment layer of illuminated letters [10]. In Ref. [11] the detection of underdrawings beneath painted layers is also reported by another group. As an example, in Fig.1 both the photograph (Fig. 1a) and the thermogram showing the presence of texts below the end paper of a XVI century book are shown.
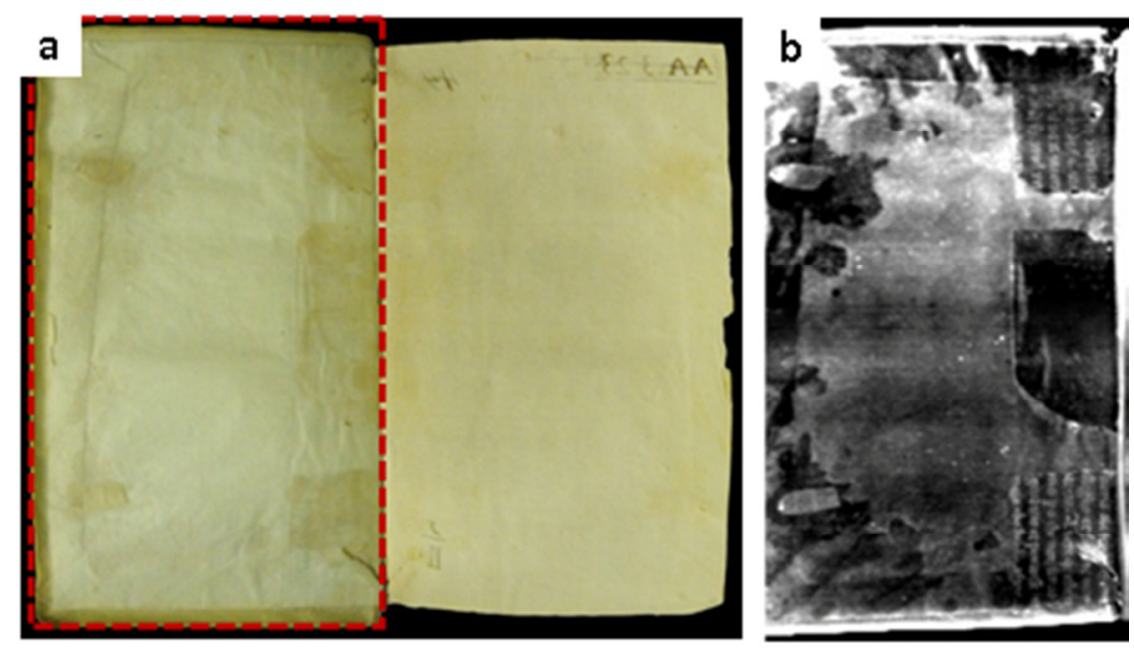

Fig. 1 XVI century paper manuscript, Biblioteca Angelica di Roma: (a) Photograph of the front end paper; (b) thermogram of the part outlined by the dashed frame in a . 
Since the presence of a thin ink layer is not expected to alter significantly the heat diffusion rate through the sample, the thermal contrast is likely to be originated by difference in the VIS light absorption and in the IR emission properties of the drawing with respect to the surrounding medium. A simple theoretical model [12] is here presented able to describe the main mechanisms and parameters which allow the detection of drawings buried into optically translucent materials by means of IRT. In this kind of samples, the IR emission results from the superposition of the IR radiation arising from different depths and, consequently, the contribution of the subsurface feature to the IRT signal depends on the temperature variation at the feature position. In this respect, the crucial role played by the effective penetration depth of the heating VIS light in determining the temperature distribution below the sample surface is examined. The validity of the proposed model is then demonstrated by its successful application to the interpretation of experimental results obtained on specifically designed test samples mimicking situations of texts buried beneath end papers in books or underdrawings laying beneath paint layers.

Finally a comparison of the results obtained by IRT and IRR in the same MWIR range is presented and discussed. It is shown that, among other, IRT can depth resolve the position of the investigated features and that it can have access to greater depths with respect to IRR, this being particularly true when the features are buried beneath highly IR diffusing materials like paper or parchment. As an example, in Fig 2 the IRT (Fig. 2a) and the IRR (Fig. 2b) images of a graphite drawing covered on the left size by different papers and on the right size by different pigment layer are shown. While IRT allows the recovery of the buried drawing features over the entire range of applied over layers, IRR can recover only the drawings covered by the paint layers.
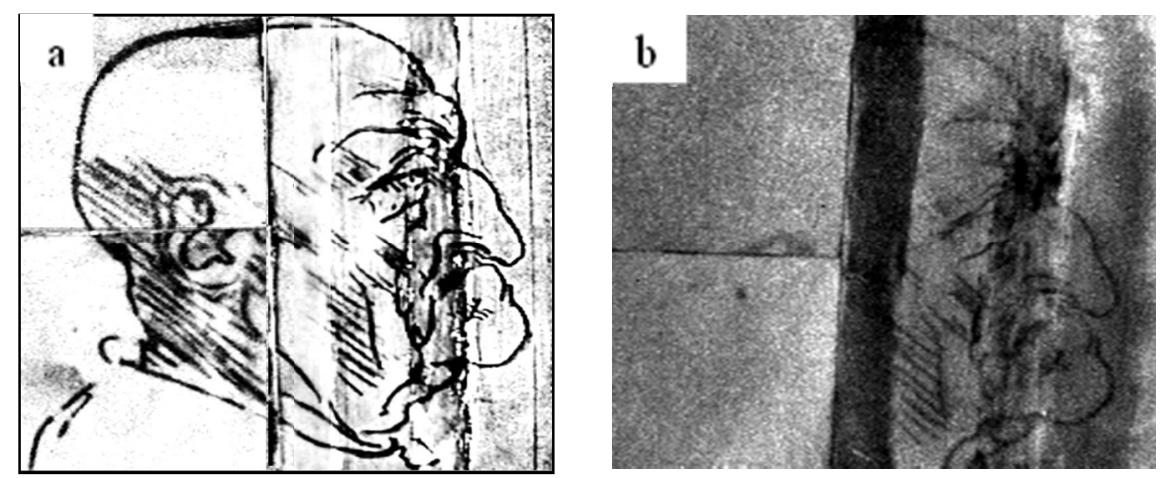

Fig.2 Pulsed IRT thermogram (a) and IRR image (b) of a test sample

\section{REFERENCES}

[1] Janssens K, Dik J, Cotte M, Susini J. Photon-Based Techniques for Nondestructive Subsurface Analysis of Painted Cultural Heritage Artifacts. Acc Chem Res. 2010;43:814-25

[2] Legrand S, Vanmeert F, Van der Snickt G, Alfeld M, De Nolf W, Dik J, Koen Janssens K. Examination of historical paintings by state-of-the-art hyperspectral imaging methods: from scanning infra-red spectroscopy to computed X-ray laminography. Heritage Science 2014; 2:13

[3] van Asperen de Boer JR. Infrared Reflectography: a Method for the Examination of Paintings. Appl. Opt. 1968;7:1711-14

[4] Daffara C, Ambrosini D, Pezzati L, Paletti D. Thermal quasi-reflectography: a new imaging tool in art conservation. Opt Express. 2012;20:14746-53

[5] Gavrilov D, Maev RG, Almond DP. A review of imaging methods in analysis of works of art: Thermographic imaging method in art analysis. Can J. Phys 2014; 92: 341-364

[6] Mercuri F, Zammit U, Orazi N, Paoloni S, Marinelli M, Scudieri F. Active infrared thermography applied to the investigation of art and historic artefacts. J. Therm. Anal. Calorim. 2011; 104:475-485

[7] Blessley K, Young C, Nunn J, Coddington J, Shepard S. The Feasibility of Flash Thermography for the Examination and Conservation of Works of Art. Stud Conserv 2010; 55: 107-120

[8] Maldague X. Nondestructive evaluation of materials by infrared thermography. London: Springer; 1993

[9] Mercuri F, R.Gnoli R, Paoloni S, Orazi N, Zammit U, Cicero C, Marinelli M, Scudieri F. Hidden text detection by infrared thermography. Restaurator 2013; 34: 195-211

[10] Mercuri F, Buonora P, Cicero C , Helas P, Manzari F, Marinelli M, Orazi N, Paoloni S, Pasqualucci A, Pinzari , Romani M, Terrei A, Verdi O, Verona Rinati G, Zammit U. Metastructure of illuminations by infrared thermography.J Cult Herit. 2018;31:53-62

[11] Ibarra-Castanedo C, Sfarra S, Ambrosini D, Paoloetti D, Benada A, Maldague X. Diagnostics of panel paintings using holographic interferometry and pulsed thermography. QIRT Journal, 2010;7:85-114

[12] Mercuri F, Paoloni S, Cicero C, Zammit U, Orazi N. Infrared emission contrast for the visualization of subsurface graphical features in artworks. Infrared Phys. Technol. 2018; 89:223-230 